\title{
Espaço, tempo e espaço-tempo: metáforas, mapas e fusões ${ }^{1}$
}

\author{
Space, Time, and Space-time: metaphors, maps, and fusions ${ }^{2}$ \\ Chris Sinha ${ }^{3}$ \\ Enrique Bernárdez $z^{4}$
}

\begin{abstract}
Resumo
Cada língua possui recursos lexicais e gramaticais para especificar as relações entre eventos, objetos e falantes no espaço e no tempo. A linguagem do espaço e a linguagem do tempo estão intimamente relacionadas na maioria das línguas, senão em todas as línguas, e tem sido proposto que a linguagem do tempo é universalmente derivada da língua do espaço por meio de mapeamentos metafóricos. Contrariamos esta hipótese, com base em uma avaliação do espaço e do tempo nas línguas do mundo, embora reconhecendo que, provavelmente, as motivações cognitivas são universais para tais mapeamentos onde eles ocorrem. Enfatizamos a variabilidade linguística e cultural das relações linguísticas do espaço-tempo, e observamos que os conceitos culturais e linguísticos de tempo podem ser derivados de outros domínios conceituais além do domínio do espaço. Ressaltamos também que a motivação da estrutura linguística pela cognição (incluída pela metáfora conceitual) é sempre mediada por padrões e processos culturais.

Palavras-chave: Espaço. Tempo. Metáfora Conceptual. Variabilidade linguística e cultural.
\end{abstract}

\begin{abstract}
Every language has lexical and grammatical resources for specifying the relations between events, objects and speakers in space and time. The language of space and the language of time are closely related in most if not all languages, and it has been proposed that the language of time is universally derived from the language of space through metaphoric mapping. We challenge this hypothesis, on the basis of a review of space and time in the languages of the world, while acknowledging that there probably are universal cognitive motivations for such mappings, where they occur. We emphasize the linguistic and cultural variability of space-time linguistic relationships, and note that cultural and linguistic
\end{abstract}

${ }^{1}$ Tradução de Wany Bernardete de Araujo Sampaio, Doutora em Linguística e em Educação Escolar, Professora aposentada, Universidade Federal de Rondônia (UNIR), Email: wansamp@gmail.com

${ }^{2}$ Este artigo foi publicado originalmente em: Farzad Sharifian (ed.). 2015. The Routledge Handbook of Language and Culture. London and New York: Routledge, pp. 309-324.

${ }^{3}$ Doutor em Psicologia, Professor de Ciência Cognitiva, Universidade de Hunan, Changsha, China. Email: christopher.sinha@gmail.com

${ }^{4}$ Doutor em Linguística, Professor de Linguística Geral, Universidade Complutense de Madrid, Faculdade de Filologia, Email: ebernard@filol.ucm.es 
concepts of time may be derived from other conceptual domains than space. We also emphasize that the motivation of linguistic structure by cognition (including by conceptual metaphor) is always mediated by cultural patterns and processes.

Keywords: Space. Time. Conceptual Cultural Metaphor.

\section{Introdução}

Espaço e tempo são frequentemente considerados como domínios universais e transculturais da linguagem e do pensamento humano, e a linguagem do espaço, tempo e movimento tem sido intensamente pesquisada nas décadas recentes. O espaço é visto como a principal fonte/domínio para a estruturação linguística e conceitual do tempo, através de mapeamentos metafóricos.

Nos níveis lexical e construcional, os domínios espacial e temporal estão estreitamente relacionados. Em muitas línguas, os significados temporais são expressos por palavras e tipos de construções cujos significados primários são analisados como sendo espaciais. Estudos tipológicos têm mostrado que o mapeamento lexical espaço-tempo é amplamente generalizado (Haspelmath, 1997) e o mapeamento construcional espaço-tempo tem sido analisado em línguas tipológica e geograficamente diferentes, tais como o Inglês, (Clark, 1973; Lakoff e Johnson, 1999), o Aymara (América do Sul: Núñez e Sweetser, 2006), o Chinês (Yu, 1998, 2012) e o Wolof (Oeste da África: Moore 2006).

Estudos recentes, entretanto, também têm revelado uma grande, e algumas vezes dramática, variação inter-línguística na linguagem do espaço e do tempo, e ambos os domínios (bem como suas relações metafóricas inter-domínio) têm sido um promitente campo de testes para a pesquisa neo-whorfiana (Levinson, 2003; Boroditsky, 2001). Muitas das evidências apresentadas como suporte aos efeitos whorfianos na linguagem espacial envolvem variação inter-línguística na estrutura de referência (Frame of Reference) espacial dominante (Levinson, 2003). De modo análogo, tem-se sugerido que a principal diferença interlínguística na linguagem temporal (algumas vezes conduzindo a diferenças cognitivas não linguísticas entre falantes) consiste não na existência de metáfora espaço-tempo per si, mas em estruturas de referência orientacionais dentro das quais metáforas espaço-tempo são sistematicamente construídas e interpretadas (Radden, 2011).

A análise do mapeamento metafórico espaço-tempo em termos da teoria da metáfora conceitual e da teoria da cognição incorporada (Lakoff e Johnson $1980,1999)$ tem influenciado particularmente na proposta de que o mapeamento linguístico espaço-tempo, sendo baseado sobre processos cognitivos não linguísticos universais (Boroditsky, 2000; Casasanto e Boroditsky, 2008), pode ser considerado como universal (Fauconnier e Turner, 2008; Núñez e Cooperrider, 2013). 
Bernárdez (2013) critica, entretanto, na abordagem da metáfora conceptual, a tendência em ou negligenciar inteiramente a dimensão cultural da metáfora - olhando exclusivamente através das lentes do universalismo cognitivo - ou, quando questões culturais são abordadas, fundir variação cultural com variação linguística, uma fusão que, de acordo com Reynoso (2013), coexistiu com a fusão entre cultura e psicologia nos escritos de Sapir e Whorf.

Um razoável número de estudos recentes, contudo, desafia a universalidade do mapeamento linguístico espaço-tempo (Sinha et al. 2011; Levinson e Majid 2013) e/ou contextualizam o mapeamento linguístico espaço-tempo, situando-o em padrões mais amplos de conhecimento cultural e visão de mundo (e.g. Hurtado de Mendoza 2002; Núñez e Cornejo 2012). Descrevemos estes e outros estudos recentes em alguns detalhes, a seguir, uma vez que eles apontam como caminho para uma compreensão mais rica, mais abrangente e mais genuína a fonte cultural das variações linguística e cognitiva.

O que está em jogo, metodologicamente, em uma contextualização cultural profunda da linguagem do espaço e do tempo? Nas disciplinas de antropologia e arqueologia, a linguagem tem sido tradicionalmente classificada, juntamente com sistemas de crenças, rituais e outras práticas linguístico-comportamentais, como o fundamento da cultura simbólica, em contraste com a cultura material, o mundo humano fisicamente construído. Nessa perspectiva, espaço e tempo, como dimensões da cultura simbólica, estão articulados não apenas na gramática e no léxico, mas também em cosmologias e visões de mundo. Nas sociedades pré-históricas, históricas e contemporâneas, as questões de identidade cultural ("Quem somos nós?") são estruturadas pelas respostas para "Onde e Quando", perguntas como "De onde viemos, para onde vamos, quando o mundo começou e quando ele acabará?".

No entanto, a distinção entre cultura material e cultura simbólica tem sido um desafio crescente na antropologia contemporânea (Boivin 2008). O espaço e o tempo não são apenas domínios universais da linguagem e do pensamento; são as dimensões fundamentais que situam da ecologia humana sociocultural e cognitiva naquilo que Bourdieu (1977: 86) chamou habitus. O habitus separa - e unifica - cultura material e simbólica de maneira que são específicos para determinados culturas e sociedades. A estrutura social não apenas é incorporada, conceitual e materialmente, no espaço e no tempo; ela também é realizada através de estruturas materiais espaciais e temporais (Hornborg 2005; Hornborg e Hill 2011).

A estruturação sociocultural do espaço e do tempo é conseguida por meio de práticas que envolvem a construção e utilização de artefatos e sistemas de artefatos que misturam o material e o simbólico em diferentes escalas. Isso inclui artefatos familiares, e historicamente evoluídos, tais como bússolas, relógios, calendários e outros sistemas de intervalo de tempo com base na língua (Birth 
2012; Sinha et al. 2011). Artefatos materiais simbólicos incluem, no entanto, a construção do ambiente (tais como a arquitetura, o layout de vilas e cidades) e a paisagem naturalmente formada pela ação humana (incluindo megalíticos, terraplanagens geomorfológicas e monumentos). Os significados desses sistemas de artefatos simbólicos materiais abarcam desde a expressão de diferenciação social (gênero, categoria, clã etc.) nas dimensões espaciais e temporais; passam pelas representações arquitetônicas das crenças cosmológicas e religiosas até o ordenamento espaço-temporal das atividades normativamente organizadas por meio de artefatos que computam o tempo.

A materialidade do significado, e a significabilidade da materialidade, é não somente um tema central na antropologia contemporânea. É também central nas abordagens recentes da ciência cognitiva, que enfatiza a importância de objetos na cognição incorporada estendida (Sinha e Jensen de López 2000), e na qual cognição e comunicação estão distribuídas sobre nichos cognitivos materiais-simbólicos (Hutchins 1995; Clark 2006; Magnani 2009; Sinha 2006, 2009, 2013). A contextualização sociocultural da diversidade linguística e cognitiva em recente trabalho, juntamente com uma perspectiva teórica geral da incorporação cultural matéria-simbólica estendida, sugere, como novidade, uma perspectiva pós-whorfiana sobre as inter-relações entre língua, cognição e cultura e suas covariações.

\section{Perspectivas histórica e contextual}

É difícil, pelo menos para os falantes do Inglês, pensar e falar sobre tempo sem empregar metáforas, e muitas dessas metáforas têm como fonte o domínio do espaço e do movimento espacial. Tome-se, por exemplo, a exposição de Sir Isaac Newton, na sua Philosophiae Naturalis Principia Mathematica (1686), sobre seu entendimento teórico do tempo. Newton acreditava que, assim como o espaço, o tempo fosse absoluto e infinito: "Absoluto, real e matemático, o tempo, dentro ou fora de si mesmo e de sua própria natureza, sem referência a nada externo, flui uniformemente e, por outra denominação, pode ser chamado de duração. Relativo, aparente e comum, o tempo é qualquer medida externa sensível (precisa ou imprecisa) de duração por meio de movimento; tal medida - por exemplo, uma hora, um dia, um mês, um ano - é comumente usada em lugar do tempo real". Paradoxalmente, talvez, ao afirmar a realidade metafísica do tempo como uma dimensão independente do espaço, Newton utilizou-se de uma metáfora "passagem", do "fluxo" (ou passagem) do "Rio do Tempo" (Smart 1949).

A separação entre tempo e espaço proposta por Newton foi desafiada no início do século XX pela Teoria Especial da Relatividade (Einstein 1920). Como contemporâneo de Einstein, o matemático Minkowski (1964: 927) afirmou: "de agora em diante, o espaço em si e o tempo em si estão condenados a desaparecer 
em meras sombras, e apenas uma espécie de união dos dois preservará uma realidade independente". O estatuto ontológico do tempo permanece indefinido na física e na filosofia da ciência; mas seja qual for o seu estatuto no universo físico, não há como negar que o tempo é um aspecto fundamental da vida no mundo experiencial e fenomenológico. É importante, no entanto, tentar distinguir este aspecto temporal da experiência, o qual nós podemos assumir ser transcultural, a partir das diversas conceituações de tempo fortemente apoiadas na abordagem cultural que passamos a explorar a seguir.

Em particular, ao descrever a temporalidade na experiência, devemos evitar, tanto quanto possível (ou, pelo menos, ser cautelosos sobre) o atraente, mas específico-cultural, uso de metáforas, não apenas de "fluxo" e "passagem" (que implicam movimento "no tempo" analogamente a movimento no espaço); mas também da estática "localização no tempo". Isto porque, mesmo que o terreno experiencial do tempo seja transcultural, conceitos de tempo variam consideravelmente, e não necessariamente é o caso de que o tempo seja transculturalmente conceituado usando-se recursos conceptuais espaciais. Traçando um paralelo explícito com a teoria de Einstein, Benjamin Lee Whorf (1940) formulou o que ele chamou de "O Princípio da Relatividade Linguística" com base na sua análise dos conceitos de tempo e temporalidade em Hopi, língua nativa norte-americana. O tempo Hopi, segundo Whorf, é "nãonewtoniano", ou seja, o falante Hopi "não tem noção geral ou intuição do tempo como um continuum que flui suave, no qual tudo no universo procede na mesma velocidade, fora de um futuro, dentro de um presente, em um passado; ou, no qual, para inverter a imagem, o observador está sendo levado continuamente, no curso da duração, de um passado para um futuro" (Whorf 1950: 27).

Whorf não relatou como, ou mesmo se, ele diretamente investigou a existência de metáforas "passagem" em Hopi. Suas conclusões foram desafiadas por Malotki (1983), que fornece uma série de exemplos do que parecem (se aceitarmos suas glossas) ser metáforas "passagem", envolvendo verbos de movimento, como "vir" e "chegar". A crítica de Malotki a Whorf, por seu turno, foi criticada (Lee 1996; Leavitt 2010), entre outros motivos, por sua tendência (como Whorf, mas espelho-imagem) para a super-interpretação de seus dados (que, é necessário dizer, é muito mais compreensível do que o publicado por Whorf).

Independentemente das especificidades da sua análise do tempo Hopi, a afirmação de Whorf de que "o tempo newtoniano" não é um modelo cultural universal recebeu suporte de pesquisas posteriores, algumas das quais são analisados a seguir. Podemos, no entanto, postular algumas categorias analíticas gerais válidas que podem servir como base para uma análise comparativa do tempo entre as línguas e culturas? O tempo como experienciado é composto das propriedades de eventos, os quais têm dois aspectos perceptíveis básicos: duração e sucessão (ou ordem sequencial). Podemos tomar como ponto de partida, 
então, a hipótese aparentemente plausível que em todas as culturas as pessoas experimentam, e são capazes de falar sobre, eventos e relações inter-eventos em termos de duração e sucessão; mas que as palavras e conceitos particulares que denotam duração temporal e marcas temporais - embora possam ser baseadas em experiências humanas universais, como a conscientização sobre o ciclo diurno - são baseados em tradições culturais e civilizacionais específicas e, por extensão, são específicos da língua e da cultura. Vamos discutir, mais tarde, que as consequências dessa variabilidade podem ser a presença ou ausência de mapeamento linguístico metafórico de espaço-tempo.

\section{Duração: conceitos culturais de intervalos de tempo}

A conceituação cultural e a expressão linguística de intervalos de tempo (isto é, conceitos lexicalizados de intervalos de duração temporal) são conhecidas por serem amplamente variáveis culturalmente. Muitas pesquisas em linguística antropológica têm abordado a variabilidade nos sistemas calendáricos (ou quase-calendáricos) e nas práticas sociais de "contagem de tempo" (EvansPritchard 1939, 1940) que eles permitem. Sistemas calendáricos verdadeiros são quantificacionais, no sentido de se basearem em um sistema de medição e, portanto, podem ser considerados como baseados em tempo, segmentando e mensurando a duração temporal na abstrata e reificada "quarta dimensão newtoniana" que Sinha et al. (2011) rotularam por "Tempo como tal". Intervalos de tempo com base no tempo, tais como a "hora" e "semana", compõem o que muitas vezes é referido como "Tempo Relógio" e "Tempo Calendário" (Levine 1997; Postill 2002).

Intervalos de tempo com base no tempo podem ser distinguidos a partir de intervalos de tempo com base em eventos. Intervalos de tempo são baseados no tempo cronológico (cf. grego antigo chronos $=$ tempo), métrico e referenciado a uma medida objetiva de "tempo decorrido", enquanto que os intervalos de tempo com base em eventos são momentâneos (kairotic, cf. antigo grego kairós $=[0$ certo] momento; Nascimento, 2012), de natureza qualitativa e normativa, não métricos e referenciados a "acontecimentos" (incluindo atividades). Intervalos de tempo com base em eventos são intervalos cujos limites são constituídos pelo próprio evento. Nesse sentido, não há diferenciação cognitiva entre o intervalo de tempo e a duração do evento ou atividade que define, e a partir da qual deriva, em geral, a lexicalização dos intervalos de tempo. O evento de referência é muitas vezes natural (como 'primavera', por exemplo: "vamos tirar umas férias na primavera"), mas às vezes convencional (como "pausa para o café", por exemplo, "vamos discutir isso durante a pausa para o café"). O intervalo de tempo baseado em eventos pode ser caracterizado como uma mudança de estado (por exemplo, "nascer do sol"), como um atributo de um evento estativo (um exemplo da língua Amondawa, da Amazônia, discutido abaixo: a palavra ara significa 'luz do dia'); ou como uma atividade cuja lexicalização pode ser 
metonímica, como em Amondawa pojiwete, "quando começamos o trabalho".

Em alguns casos, os termos para intervalos de tempo baseado em eventos derivam de termos que se referem tanto à atividade como ao lugar (ou mudança de lugar). Por exemplo, no Inglês moderno, o termo "enquanto", que coordena a duração dos eventos, deriva do antigo termo germânico que significa "descanso" e está relacionado com o islandês moderno hvila, que significa tanto "descanso" como "cama" (lugar de descanso ), enquanto o antigo cognato Norse de "descanso", rost, significava "a duração de uma viagem entre dois pontos" e mais tarde adquiriu um significado espacial mais preciso para indicar uma distância de $12 \mathrm{~km}$. A polissemia de significado temporal e espacial neste e em outros exemplos é mais bem compreendida como envolvendo uma fusão metonímica do que como um mapeamento metafórico, questão a que voltaremos abaixo.

Embora intervalos de tempo com base no tempo sejam baseados em ciclos naturais (astronômicos) de eventos, suas divisões são convencionais e a medição da duração temporal é obtida pela contagem num sistema numérico. A terminologia para contar ou falar sobre o tempo tem as suas raízes etimológicas em palavras germânicas para contar (e recontar ou dizer) (por exemplo, no holandês moderno rekenen, "contar"). Vale ressaltar que a raiz reg- do Indoeuropeu, que é a origem de reckon, rekenen etc., significava "mover-se em uma linha reta" (um cognato, em Inglês, é row), uma etimologia que atesta a importância do caminho e do movimento na conceituação de número e de linha numérica (Lakoff e Núñez 2000).

Sistemas calendáricos geralmente possuem uma estrutura recursiva de tal modo que os diferentes intervalos de tempo são encaixados uns dentro dos outros, e/ou uma estrutura de intervalos metricamente sobrepostos. Esses intervalos são de natureza tipicamente cíclica, com ambos os ciclos incorporados e sobrepostos. O mais familiar para nós é o calendário gregoriano lunar e solar (mais estritamente, mensal e anual) agora adotado internacionalmente. Um exemplo da complexidade que os sistemas de calendários baseados numericamente podem atingir é fornecido pela civilização clássica Maia, da América Central, que usou três diferentes sistemas de calendário: (i) o Contagem Longa poderia ser usado para especificar qualquer dia na história Maia e poderia (em princípio) gerar referências de tempo em uma escala infinita, um fato que estruturou a cosmologia Maia e foi a principal motivação e função para o conhecimento matemático Maia; este sistema funcionava com valor local e o número zero, ambos desconhecido para a antiguidade clássica mediterrânea; (ii) o calendário Tzolkin (que conta os dias ou Ano Sagrado) era um calendário cerimonial, com 20 períodos de 13 dias, completando assim um ciclo ritual a cada 260 dias; (iii) o Haab era um calendário civil com base em um ano de 360 dias, com 18 períodos de 20 dias. Cinco dias foram adicionados no final do ano Haab para sincronizá-lo, aproximadamente, com o ano solar (Edmonson, 1976; 
Wright, 1991). O ciclo ritual de 260 dias não era exclusivo para os Maia e, possivelmente, não se originou com eles: um pouco anteriormente, o Zapoteca clássico, da América Central, também empregou esse intervalo de tempo (Flannery e Marcus, 2012: 371).

Sistemas de calendários não são meramente "cronometristas"; eles são expressão de crenças e valores culturais. $\mathrm{O}$ sistema de calendário gregoriano, por exemplo, sobrepõe, conceitualmente, sua estrutura cíclica a um modelo linear de tempo (Bernárdez, 2003), que envolve o movimento a partir de uma origem (do nascimento de Cristo) para um fim de linha nocional (fim dos dias). Essa dualista conceituação cíclica-linear (com diferentes relações de dominação entre ciclicidade e linearidade) também é característica de outros sistemas de calendários, como o maia, o islâmico e o védico (Keyes, 1975). Schieffelin (2002) documenta as mudanças linguísticas que ocorrem em Bosavi Kaluli (Papua, Nova Guiné), envolvendo tanto o apagamento como a inovação no léxico, na gramática e nos gêneros discursivos, em consequência da introdução de conceitos cristãos de tempo, por missionários, e do calendário gregoriano, por agências governamentais.

Um estudo clássico sobre intervalos de tempo com base em eventos sistemáticos, porém não quantificacionais, foi realizado por Evans-Pritchard $(1939,1940)$, que descreveu o que ele chamou "relógio do gado" ou "tempo de trabalho" Nuer. O tempo na sociedade Nuer, segundo ele, se baseava em mudanças ambientais associadas a atividades. O conceito de tempo na sociedade Nuer foi, portanto, um produto da interação entre "tempo ecológico" e "tempo da estrutura social". Embora houvesse nomes para (aproximadamente) meses lunares, a sociedade Nuer, conforme descrito por Evans-Pritchard, não contava ou media o "Tempo como tal"; a língua não tem uma palavra, quer para a noção abstrata de tempo, ou por unidades de tempo abstrato, e pontos de referência temporais são fornecidos por atividades sociais.

O Nuer não tem nenhum sistema numérico abstrato de contagem do tempo com base em observações astronômicas, mas apenas divisões descritivas de ciclos de atividades humanas... uma vez que os meses estão vinculados ao ecológico [sic] e o processo social do calendário é um esquema conceitual que permite ao Nuer visualizar o ano como uma sucessão ordenada de mudanças e calcular, em certa medida, a relação entre um evento e outro em símbolos numéricos abstratos. (Evans-Pritchard 1939: 197, 200)

Os meses Nuer não eram estritamente lunares, nem baseados em qualquer outro número fixo de dias. Em vez disso, eram convencionados com base em ambos os ciclos - lunar e ecológico -, associados ao ritmo das atividades sociais. Em resumo, o tempo, para o Nuer, consistiu em uma relação esquematizada entre eventos sociais e ambientais definidos, e a contagem do tempo Nuer não era um 
cálculo rigoroso de, ou em, "Tempo como tal", mas apenas uma estimativa, raramente expressa numericamente, com base nas relações da estrutura social e atividades.

O tempo Nuer não é o único sistema de intervalos de tempo relatado na literatura antropológica que emprega aproximadamente meses lunares especificados em um sistema não quantificado. O sistema de intervalo de tempo Ainu, uma cultura do sul de Sakhalin, que em outros aspectos (economia, estrutura social e tempo cosmológico) é bastante diferente do sistema Nuer, inclui meses lunares que regulam os rituais, bem como a atividade de caça e pesca. No entanto, "os Ainu são bastante alheios a nomes dos meses, bem como ao número de meses do ano" (Ohnuki-Tierney 1973: 289), e os Ainu, cujo sistema básico de número (números não derivados) se estende a cinco, raramente ou nunca contam numericamente intervalos de tempo, usando a oposição entre dois ou três e o número derivado seis para contrastar durações curtas e longas.

Enquanto o sistema de intervalos de tempo Nuer, baseado em eventos, pode ser pensado como quase-calendárico, permitindo práticas de contagem de tempo estimado, os meses lunares Ainu, não nomeados, não participam de qualquer coisa parecida com um calendário anual. Ohnuki-Tierney conclui que "o conceito Ainu de tempo é basicamente qualitativo; medição quantitativa de tempo é pouco desenvolvida. Por conseguinte, não há divisões temporais que representem unidades mensuráveis; eles se distinguem de outras unidades na mesma escala de tempo pelo significado especial que os Ainu atribuem a eles" (op. cit. p. 292).

Uma conjunção diferente entre um pequeno sistema de números (quatro números) e um sistema de intervalo de tempo não-quantificacional é encontrado em Amondawa, uma língua Tupí-Guaraní da Amazônia Ocidental (Sinha et al. 2011; Silva Sinha et al. 2012). O sistema de intervalo de tempo Amondawa é não-calendárico, no sentido de que não há nomes para mês, semana e ano; intervalos de tempo maiores são baseados em estações e suas subdivisões (oito termos, ao todo) e os menores se baseiam sobre os segmentos do dia.

Amondawa não é um caso único. A análise preliminar dos dados coletados por Sampaio e Silva (2012) sugere que outras línguas amazônicas, geneticamente relacionadas e não relacionadas, possuem um perfil semelhante. Além disso, uma língua completamente não relacionada, a língua isolada Yélî Dnye, falada na ilha Rossel, 450 quilômetros ao largo de Papua Nova Guiné, também não tem termos de calendários, entretanto (como em Ainu) o termo para a "lua" pode designar um mês lunar aproximadamente. O Yélî Dnye também tem quatro termos sazonais e termos contrastantes para dia e noite. Levinson e Majid (2013: 3) afirmam que "isso parece esgotar as unidades de tempo indígenas". Curiosamente, as línguas Amondawa e Yélî Dnye parecem 
não empregar construções metafóricas espaço-tempo, embora ambas revelem mapeamentos e fusões espaço-tempo lexicais. Voltaremos posteriormente a este conjunto de resultados.

\section{Esquemas relacionais temporais e metáforas espaciais}

Duração é a extensão temporal. Sucessão é a posição temporal. Ao afirmar isso, estamos, de fato, convidando a uma analogia entre duração e extensão espacial e sucessão e posição espacial (em frente/atrás, antes/ depois). Os eventos são, em alguns aspectos, como objetos; mas eles também são diferentes. Os objetos estão localizados no espaço, e resistem, embora de maneira fugaz, com o tempo. Eles têm propriedades como massa e energia. Os eventos são "localizados" no tempo, bem como no espaço ocupado pelos objetos envolvidos no evento, tendo propriedades de duração e sucessão. Além disso, nós empregamos (evento) marcas temporais para nos orientar no tempo, assim como empregamos (objeto) marcas espaciais para nos orientar no espaço. Palavras para duração temporais incluem adjetivos tais como "longo" e "curto", mas também medidas de intervalos de tempo como "10 segundos" e "quatro meses". Marcas temporais incluem advérbios, como "hoje", "ontem" e "amanhã", tempos nomeados do dia (meia-noite, três e meia), datas (01 de maio) e outros eventos calendaricamente estruturados (Páscoa, meu aniversário, data da graduação).

Enquanto marcas espaciais são empregadas a serviço da navegação literal no espaço, envolvendo movimento físico, não podemos viajar fisicamente no tempo, que não seja por ser "levada avante pelo fluxo do tempo". Nossa navegação temporal é, portanto, inteiramente realizada na mente e no discurso linguístico, através de lembranças, imaginação e aquilo a que chamamos o "planejamento para frente" das ações, eventos e os locais de ações e eventos.

A analogia entre sucessão temporal e da ordem espacial foi a base para uma distinção feita pela primeira vez pelo filósofo John McTaggart (1908) entre o que hoje designamos dois quadros de referência diferentes e esquemáticos. A denominada "A- série" de McTaggart pode ser pensada como o evento visto a partir do ponto de vista do momento presente. Como o momento presente está em constante mudança, o evento "passa" do futuro para o passado, daí a sua designação, por alguns filósofos, como tempo de "passagem". Núñez e Cooperrider (2013) referem-se a A-série temporal como D-tempo (sobre tempo dêitico, ver também Evans 2013; Le Guen e Balam 2012). D-tempo é a base esquemática do tempo gramatical, em línguas que têm tempo (verbal ou nominal) e é também o tempo de dêiticos adverbiais como "amanhã" ou "ontem" e de marcas temporais tais como "o próximo Natal".

A chamada "B-série" de McTaggart, em contraste, é não marcada por tempo gramatical na medida em que representa eventos unicamente em termos 
da sua ordenação em uma sequência de eventos, cada um dos quais podem ser marcados como "antes" ou "depois" de outros eventos, e em que nenhum evento constitui um centro dêitico privilegiado. Sinha et al. (2011) se referem a esta "B-série" como o tempo posicional, e Moore (2011) refere-se a ela como um quadro de referência "baseado em campo" (em contraste com o "A-série", que ele designa como quadro de referência "perspectiva de ego"). Por razões de coerência, usaremos a abreviatura S-tempo (sequência de tempo), de Núñez e Cooperrider. S-tempo é o tempo em que as relações são especificadas por 'antes' e 'depois', bem como 'cedo' e 'tarde', 'primeiro' e 'último'.

D-tempo e S-tempo podem ser, ambos, esquematicamente representados como linhas de tempo linear, e S-tempo como um ciclo de repetição; mas, como veremos, essas atribuições devem ser tratados com cautela, por não necessariamente possuírem a realidade psicológica de um determinado falante. O tempo calendárico é, por definição, uma representação S-tempo, mas isso não significa (contra Gell, 1992) que eventos S-tempo são intrinsecamente "datados", com referência a um calendário; eles são, sim, intrinsecamente ordenados.

Embora em um determinado idioma recursos lexicais particulares (por exemplo, palavras que correspondem a "antes" e "depois", como em Yucatec Maia (Bohnemeyer, 2002), ou os recursos gramaticais possam ser ausentes (por exemplo, tempo verbal, como em chinês), não há relatos sobre "línguas atemporais", sem expressões S-tempo e/ou D-tempo. Podemos razoavelmente supor, então, que não só todos os seres humanos normalmente desenvolvidos experienciam duração e sucessão, mas também que todas as línguas têm recursos para comunicar o tempo de um evento determinado em relação a outro evento (S-tempo) ou ao tempo da expressão oral (D-tempo). Isso deixa aberta, porém, a questão de saber se todas as línguas codificam linguisticamente tanto as relações D-tempo como S-tempo e, se for o caso, se as línguas variam no grau em que esses dois esquemas relacionais temporais fundamentais são elaborados.

Como o próprio McTaggart observou, parece natural para interpretar D-tempo metaforicamente, em termos de passagem, ou movimento, em que tanto "dizemos que os eventos saem do futuro [ou] que nós mesmos avançamos para o futuro." (McTaggart 1908, 470, n.1). Estes dois esquemas metafóricos, envolvendo tanto o movimento de um evento em relação ao "agora" de um experimentador ou o movimento de um experimentador em relação a um marco temporal, foram apontados por Clark (1973) como a metáfora "tempo em movimento" (MT) e a metáfora "ego em movimento" (ME). Eles são exemplificados por expressões como "as férias estão chegando" (MT), "ela está vindo até seus exames" (ME), "o prazo já passou” (MT).

Construções MT e ME são, ambas, casos particulares de uma classe mais geral de metáforas de "passagem" D-tempo, cujo domínio de origem é o 
movimento espacial. As Metáforas de "passagem" podem, mas não precisam, envolver o uso explícito de verbos de movimento, tais como vir, ir, passar, chegar e assim por diante; em vez disso, elas podem ter sua interpretação metafórica em locuções adverbiais como "o próximo ano", e podem ser estativas e de orientação, bem como dinâmicas, por vezes envolvendo movimento fictício (Talmy, 1999): por exemplo, "minha infância está atrás de mim", "ele enfrenta uma pena de prisão grave". Metáforas conceituais, como a conhecida "a vida é uma viagem" (Lakoff e Johnson, 1980) podem ser consideradas como esquemas generalizados derivados de metáforas de "passagem", estruturados de forma semelhante, uma interpretação que difere da visão de construções de "passagem" especificamente como instanciações de metáforas conceituais préexistentes e transculturais.

S-tempo, bem como D-tempo, pode ser conceitualizado metaforicamente em termos de relações espaciais, com a diferença de que as relações são entre eventos, preferivelmente do que entre um experimentador e um evento, ainda que os mesmos termos espaciais empregados também possam ser usados em metáforas de "passagem". Assim, podemos dizer "o check-in está à frente do embarque", ou "além do primeiro rito de passagem estão muitos mais testes". S-tempo é sempre posicional em significado, isto é, as relações inter-eventos de antecedência temporal e sucessão são invariáveis, ao passo que as relações de passado e futuro são uma constante mudança em relação ao momento presente. Propomos, então, chamar de metáforas espaciais as metáforas posicionais S-tempo.

S-tempo é frequentemente dito ser conceitualizado de uma perspectiva externa, mas este não é necessariamente o caso, como pode ser visto a partir de alguns dos exemplos apresentados, os quais são ancorados ao ponto de vista de um falante ou experimentador a partir de uma posição dentro de uma linha do tempo. S-tempo também é muitas vezes visto como estativo, com eventos sequenciados como as contas em um colar; mas verbos de movimento também podem ser empregados em metáforas posicionais, por exemplo, "o cocktail vem à frente do buffet" ou "questões seguirão a palestra".

Como esses exemplos sugerem, enquanto as expressões não metafóricas de S-tempo (como "check-in pelo menos 40 minutos mais cedo do que o embarque") não implicam uma perspectiva do falante, as metáforas posicionais tais como "check-in pelo menos 40 minutos antes do embarque", como expressões espaciais que envolvem, por exemplo, relações dêiticas frente/atrás, implicam tal perspectiva. A existência de perspectiva, então, é comum às metáforas espaçotempo, tanto de "passagem" como posicionais, embora apenas metáforas de "passagem" expressem relações temporais dêiticas (D-tempo). Além disso, algumas metáforas posicionais que dependem de movimento fictício, como "check-in antes do embarque", parecem ser esquematicamente derivadas de uma metáfora de "passagem" ME. A diferença entre metáforas de "passagem" 
e metáforas posicionais é o contraste semântico entre a subjacente relatividade (D-tempo) versus a invariância (S-tempo) da relação temporal conceitualizada.

\section{A assimetria de D-tempo e S-tempo na perspectiva inter- línguística}

De nosso conhecimento, não há relatos de qualquer língua que careça de recursos lexicais para a marcação D-tempo, embora a sua gramaticalização não seja universal. Existem línguas que não marcam o tempo gramatical no verbo e há línguas em que D-tempo é marcado no substantivo (Nordlinger e Sadler, 2004) em vez de tempo verbal; mas todos os idiomas parecem ter pelo menos um repertório de dêiticos adverbiais que indicam gradações de passado e futuro de eventos com respeito ao tempo do enunciado. Sistemas lexicais D-tempo podem ser de considerável complexidade: em Yéli Dnye, por exemplo, há termos ordinais monolexêmicos muito específicos para designar os dias de "anteontem" e para "o $10^{\circ}$ dia, no futuro" e um sistema produtivo especificando os dias ainda mais no futuro; seu sistema de tempo também faz referência ao dia específico do evento (Levinson e Majid, 2013: 2). O sistema ordinal de contagem de dias em Yéli Dnye é particularmente interessante porque, embora a língua careça inteiramente de um calendário, o sistema de contagem de dias é posicional e, assim, pode-se dizer que compartilha ou mistura propriedades de S-tempo com as de D-tempo.

Não temos conhecimento de quaisquer línguas em que relações inter-eventos S-tempo são gramaticalizadas separadamente do sistema temporal, que, embora seja deiticamente ancorado ao tempo da enunciação, também pode especificar sequência, por exemplo, "Ela tinha saído quando ele chegou"; por vezes em conjunto com os termos antes/depois, por exemplo, "Ele chegou depois que ela tinha deixado".

S-tempo é inter-linguisticamente mais variável do que D-tempo na expressão lexical e conceitualização. Consequentemente, é mais difícil estabelecer se sua lexicalização é comum a todas as línguas. Como notado anteriormente, apesar das reivindicações pela universalidade de conceitos lexicais "antes" e "depois" (Goddard 2010), nem todas as línguas possuem esses termos (Bohnemeyer 2002).

Embora não conheçamos línguas relatadas como tendo ausência de lexemas que podem ser glossados como "cedo" e "tarde", em muitos casos, estes lexemas são advérbios dêiticos e não é claro se os significados S-tempo "mais cedo [do que o evento]" e "mais tarde [do que o evento]" são analiticamente ou discursivamente distinguíveis dos significados D-tempo "mais cedo" e "mais tarde" [do que agora]. Por exemplo, a língua papua Mian tem um termo sino, derivado de $\sin$ "velho", que é glossado como "antigamente, antes, mais cedo" (Fedden e Boroditsky, 2012: 5). De modo mais geral, podemos dizer que, tanto 
nas expressões não metafóricas como nas metafóricas, as mesmas palavras e construções podem ser usadas para expressar as relações tanto D-tempo como S-tempo.

Mesmo que uma língua careça de marcas de temporalidade e de léxico equivalente a antes/depois, os falantes são capazes de empregar outros recursos gramaticais para expressar relações temporais S-tempo inter-eventos. Por exemplo, os falantes de Yucateca Maia empregam marcadores aspectuais completivos e outros para comunicar sequência temporal, em conjunto com o espelhamento icônico da ordem de menção dos acontecimentos na ordem de sua ocorrência (Bohnemeyer 2009; Le Guen e Balam 2012). Assim, isto poderia ser considerado como "lacuna" na restrição da gramática e do léxico, mas não impede a conceituação e expressão de S-tempo. Este ponto é ainda reforçado quando consideramos a universalidade da narrativa (que, por definição, envolve a representação das sequências de eventos) entre diferentes culturas, como um artefato linguístico muitas vezes também representado por outro significado como, por exemplo, o significado pictórico. Na verdade, os artefatos linguísticos pictóricos e materiais-simbólicos podem ser considerados como importante e, em alguns casos, o principal - meio que permite a expressão de conceitos S-tempo culturalmente significativos.

Embora representações pictóricas ou outras representações materiaissimbólicas de S-tempo possam estar ausentes (como em Amondawa e Yélî Dnye), S-tempo pode ainda ser representado por sistemas simbólicos transmitidos linguisticamente. Não só os sistemas de calendários, mas sistemas não-calendáricos de intervalo de tempo sazonal e diurno podem ser considerados como artefatos de S-tempo específicos da cultura. Sistemas de parentesco, memória genealógica e alguns sistemas onomásticos também envolvem claramente sequência temporal. Um exemplo flagrante deste último é o sistema onomástico Amondawa, em que os indivíduos mudam seus nomes nos pontos de transição em sua "passagem" através de diferentes fases da vida, delineado a partir de um inventário estruturado por gênero e metade exogâmica a que pertencem, bem como as fases da vida denominadas (Silva Sinha et al. 2012).

Concluímos, a partir deste breve estudo que, embora os esquemas, tanto D-tempo como S-tempo, estejam - quase certamente - transculturalmente presentes, há uma variação considerável nas formas específicas em que estes são organizados e expressos em diferentes línguas. Tem havido muito mais pesquisa linguística sobre D-tempo - porque é fundamental para sistemas de tempo gramatical - do que sobre S-tempo, que não é convencionalmente gramaticalizado separadamente de D-tempo, e que é expresso tanto no léxico como nos constituintes simbólico-culturais em um nível mais alto do que palavras individuais ou sentenças (narrativas, intervalo de tempo e sistemas de parentesco), muitas vezes envolvendo outros recursos semióticos (por exemplo, 
pictóricos) além da língua. As investigações sobre esses sistemas artefatuais têm sido mantidas mais na antropologia cultural e na psicologia do que na linguística. A representação S-tempo, sugerimos, é mais variável culturalmente do que a representação D-tempo. Além disso, conforme discutiremos, ela é o meio específico de mediação e representação de S-tempo, com o qual parece estar correlacionada e, possivelmente ligada causalmente, à existência de metáforas de "passagem" e posicionais espaço-tempo.

\section{Espacialização do tempo no mundo}

Como já mencionado, vários estudos recentes investigaram se as diferenças inter-linguísticas nos padrões de mapeamento espaço-tempo envolvem correlações entre os quadros de referência temporais e espaciais. Assim como nas análises linguísticas, duas metodologias que têm sido frequentemente utilizadas nesses e em outros estudos são a gravação de gestos que acompanham a fala e o posicionamento de falantes elicitado por meio de figuras ou outros símbolos materiais que representem eventos temporalmente ordenados. No momento da escrita, não emergiram imagens inter-linguisticamente consistentes destes estudos. Algumas investigações encontraram isomorfismos linguísticos e não linguísticos sistemáticos entre os quadros de referência espaciais e temporais (Gaby, 2012; Núñez et al, 2012). Outros não encontraram tal relação, e/ou encontraram padrões de resposta sugestivos de que os falantes nativos não constituem uma amostra uniforme, sendo o contato linguístico e a experiência educacional mediadores variáveis independentes (Brown, 2012; Le Guen e Balam, 2012; Levinson e Majid, 2013).

Com base nas suas investigações em Tzeltal e Yélî Dnye, respectivamente, Brown (2012: 10) conclui que "não há transferência automática de quadros de referência espaciais para os temporais"; e Levinson e Majid (2013: 10) dizem que seu estudo "lança dúvidas sobre a forte tendência universal para o mapeamento sistemático do espaço-tempo". Le Guen e Balam (2012: 14) investigaram, em um estudo multi-metodológico, D-tempo e S-tempo em Yucatec Maia, encontrando que nenhum deles é mapeado por uma linha do tempo metafórica. Eles relatam que S-tempo é conceitualizado como "uma sucessão de eventos concluídos, não espacialmente organizados", na qual se atribui "ciclicidade" tanto a eventos individuais como a sequências de eventos. Eles concluíram que "a utilização de um quadro de referência geocêntrico [espacial] em vez de fornecer um modo de mapeamento de tempo para espaço, evita-o e só permite um mapeamento espaço-tempo que se opõe a tempo corrente e remoto (passado e futuro)".

É importante notar, no entanto, que em nenhum contexto cultural até agora investigado os participantes não conseguiram entender, em pelo menos uma tarefa, a atividade de representar sequência temporal, produzindo um layout posicional espacialmente ordenado, mesmo em casos onde o mapeamento 
sistemático espaço-tempo - seja linguístico, gestual ou artefactual - foi encontrado como ausente (Figura 1). Isso apoia a hipótese de que o pré-requisito capacidade cognitiva ou tendência para o mapeamento do espaço-tempo é um universal humano transcultural. Deve-se igualmente notar, no entanto, que isto não significa, de modo algum, que todas estas representações espaciais de S-tempo sejam retilíneas; frequentemente elas são linhas curvas, e o mesmo vale para representações espaciais D-tempo, as quais não podem ser organizadas com assimetria linear (Le Guen e Balam 2012).

Em outras palavras, mesmo se o mapeamento espaço-tempo repousa sobre fundamentos cognitivos universais, não há nada de universal sobre a noção de uma "linha do tempo". Com toda a probabilidade, o conceito linear de "linha do tempo" tem se desenvolvido historicamente em sinergia com o conceito de métrica numérica linear, a qual também foi mostrada não ser um universalmente transcultural (Dehaene et al. 2008).

Figura 1 - Amondawa mapeando espacialmente os intervalos de tempo (estações do ano).

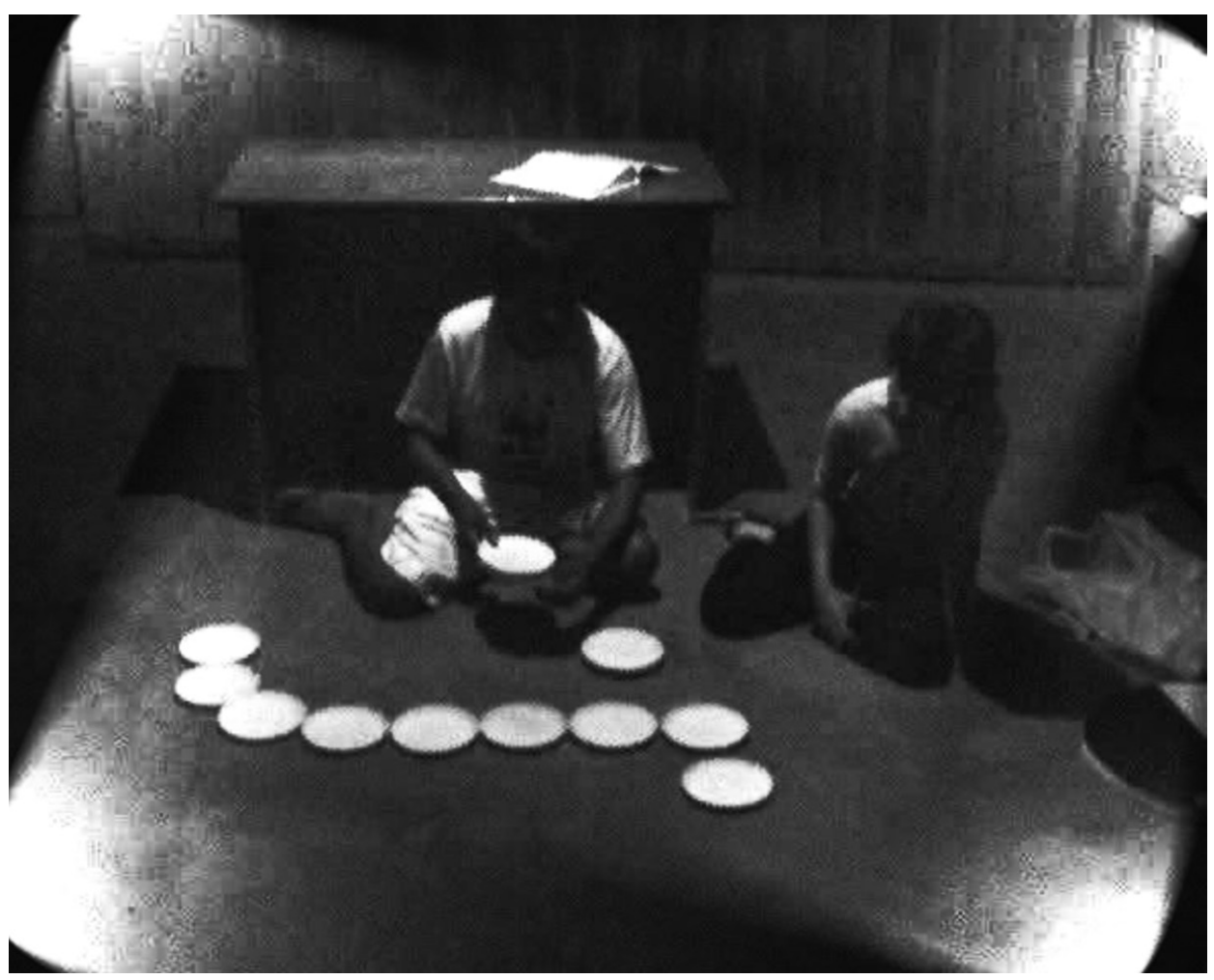

Ao avaliar a alegação de que a espacialização do tempo é universal, precisamos distinguir (1) mapeamentos espaço-tempo ocasionais e não sistemáticos, de correspondências e fusões espaço-tempo, (2) mapeamentos 
sistemáticos envolvendo a transposição de um quadro de referência espacial para D-tempo e/ou S-tempo; e/ou o recrutamento generalizado de construções de movimento e localização espaciais em metáforas de "passagem" ou metáforas posicionais. Parece provável que muitas, talvez todas, as línguas manifestem ocorrências de (1) sem (2) (este é o caso, por exemplo, de Amondawa e Yélî Dnye, que carecem de metáfora espaço-tempo sistemática).

Mapeamentos não sistemáticos podem ser metafóricos, metonímicos ou fusionais. Um exemplo de mapeamento metonímico é a palavra Amondawa que significa "ao longo do caminho do sol", fornecido por um falante para a tradução de "hoje" (Sampaio e Silva Sinha, 2012). Deve-se notar que ainda não sabemos se este é um uso arraigado, ou se é uma cunhagem relativamente recente; mas serve para demonstrar que a ausência de mapeamento sistemático espaço-tempo não implica a ausência completa de produção de mapeamento espaço-tempo, especialmente no nível lexical. Em outros casos, como o uso dos mesmos lexemas (por exemplo, 'longo') para a extensão espacial e temporal, ou como rost, do antigo Norse, discutido acima, que tem significado tanto de distância como de duração, o "mapeamento" é indiscutivelmente uma fusão polissêmica. O mesmo caráter fusional pode ser visto, em muitas línguas, no uso do mesmo termo ou gesto para "aqui" e "agora", com significado tanto espacial como temporal; ou seja, um centro dêitico unificado espaço-temporal. Nenhum desses mapeamentos metonímicos e/ou fusões espaço-tempo não sistemáticas, a nosso ver, contrariam o proposto por Núñez e Cooperrider (2013), facilmente classificável como "interpretações espaciais de tempo".

A noção de "fusão" nos leva a uma reflexão sobre a oposição entre a visão newtoniana de espaço e tempo como domínios distintos e o domínio singular do espaço-tempo introduzido no pensamento ocidental por Einstein, e atribuído por Whorf à língua e pensamento Hopi. Há extensa documentação da lexicalização de um unificado, ou fundido, conceito de espaço-tempo em pelo menos duas línguas, Aymara e Quechua. Aymara e Quechua são línguas andinas filogeneticamente não relacionadas (Adelaar, 2004) que compartilham conceptualizações e práticas culturais e vocabulário, especialmente no domínio do espaço-tempo. Em ambas as línguas, o espaço-tempo é chamado pacha, a partir do qual derivam os termos Aymara pachamama (Mãe Terra), akapacha (este tempo e época) (Núñez e Cornejo, 2012) e muitos outros compostos.

Os termos Aymara nayra, "frente" e qhipa, 'voltar', correspondem, respectivamente, aos cognatos Quechua ñawpaq e qhipa. Núñez e Sweetser (2006) mostraram que os falantes Aymara empregam uma linha de tempo D-tempo ancorada deiticamente, na qual o futuro é conceituado como estar à frente do falante/experienciador, e o passado está atrás dele. Essa "reversão" da direcionalidade do esquema ocidental, em que o futuro é pensado como para frente e o passado para trás, se manifesta não só no discurso, mas também em gestos compartilhados com a fala em Aymara. O Quechua emprega o que parece 
ser uma linha de tempo D-tempo idêntica ao Aymara (Hurtado de Mendoza, 2002); embora Faller e Cuéllar (2003) contestem isso, argumentando que as palavras Quechua ñawpaq e qhipa de fato não correspondem a "passado" e "futuro", mas são expressões S-tempo correspondentes a "mais cedo que" e "mais tarde que". De fato, isso caracteriza adequadamente a sua utilização no manuscrito Huarochirí, do Século 17, (Arguedas, 2011), analisado por Bernárdez (2013); mas devemos notar que termos relacionais temporais podem ser usados com significados tanto D-tempo como $\mathrm{S}$-tempo em várias línguas; os termos Aymara equivalentes parecem ser assim usados.

Uma análise aprofundada do uso espacial de nayra e qhipa em Aymara, por Núñez e Cornejo (2012) revelou que, curiosamente, o uso de termos está situado em um quadro de referência absoluto em que nayra corresponde a Leste e qhipa corresponde a Ocidente. Esta esquematização foi transferida pelo povo Aymara à variedade andina do espanhol e pode levar a situações como aquela em que um falante pode dizer que ele está qhipa (Aymara) ou atrás de (espanhol 'atrás de') alguém sentado diretamente de frente para ele (Núñez e Cornejo, 2012: 972). Núñez e Cornejo também analisaram como essas correspondências, que implicam a visibilidade, bem como a direção cardeal, são expressas ou materializadas no ambiente construído. Não temos espaço aqui para entrar em detalhes deste e de outros estudos, mas um resumo do complexo de correspondências que caracterizam pacha, o espaço-tempo andino, é apresentado no Quadro 1.

Quadro 1 - Correspondências de espaço-tempo nas línguas andinas não relacionadas geneticamente Aymara (Nuñez e Cornejo, 2012) e Quechua (inter alia Hurtado de Mendoza 2002; Bernárdez, in press).

\begin{tabular}{|c|c|c|c|c|c|c|c|}
\hline Língua & $\begin{array}{c}\text { Termo } \\
\text { Lexical }\end{array}$ & Glossas & $\begin{array}{l}\text { Significado } \\
\text { Espacial } \\
\text { relational }\end{array}$ & $\begin{array}{c}\text { Direção } \\
\text { Cardinal } \\
\text { Associada }\end{array}$ & $\begin{array}{l}\text { Significado } \\
\text { S-time } \\
\text { Associado }\end{array}$ & $\begin{array}{l}\text { Significado } \\
\text { D-time } \\
\text { Associado }\end{array}$ & $\begin{array}{l}\text { Visibili- } \\
\text { dade }\end{array}$ \\
\hline \multirow[t]{2}{*}{ Aymara } & nayra & $\begin{array}{c}\text { face frente } \\
\text { olho }\end{array}$ & $\begin{array}{l}\text { em frente } \\
\text { de }\end{array}$ & Leste & $\begin{array}{c}\text { mais cedo } \\
\text { [antes] }\end{array}$ & Passado & Visível \\
\hline & qhipa & back & atrás de & Oeste & $\begin{array}{c}\text { mais tarde } \\
\text { [depois] }\end{array}$ & Futuro & Invisível \\
\hline \multirow[t]{2}{*}{ Quechua } & ñawpaq & frente & $\begin{array}{c}\text { em frente } \\
\text { de }\end{array}$ & & $\begin{array}{c}\text { mais cedo } \\
\text { [antes] }\end{array}$ & Passado (?) & Visível \\
\hline & qhipa & trás & atrás de & & $\begin{array}{c}\text { mais tarde } \\
\text { [depois] } \\
\text { [seguindo] }\end{array}$ & Futuro (?) & Invisível \\
\hline
\end{tabular}

Em contraste com os mapeamentos não sistemáticos e fusões metafóricometonímicas discutidas acima, correspondências andinas espaço-tempo são altamente sistemáticas e informadas por uma visão cultural do mundo. Devemos considerar este conjunto estruturado de mapeamentos e correspondências como 
metafórico? Ou devemos seguir as línguas indígenas e seu modelo cultural compartilhado em relação às correspondências como existindo dentro de um único domínio conceptual do espaço-tempo? Nós consideramos esta última como preferível, por algumas razões. Em primeiro lugar, o testemunho dos falantes, ressaltando que o movimento no espaço é simultaneamente movimento no tempo, e negando que ñawpaq e qhipa são apenas equivalentes de "frente" e "atrás" deva ser levado a sério (ver, por exemplo, Hurtado de Mendoza, 2002:71).

Em segundo lugar, após uma investigação mais aproximada, o eixo AymaraQuechua nayra/ñawpaq- qhipa não é simplesmente a linha de tempo ocidental passado-futuro, invertida e com correspondências adicionais. A palavra para "ancestral" em Quechua é ñawpaqkuna, significando aqueles em frente, que são guias de conduta correta (Calvo Pérez, 1995: 21). Esta centralidade dos antepassados em um espaço-tempo que é também um universo moral é compartilhada por outras culturas ameríndias. Por exemplo, Basso (1988: 112) destaca o papel dos nomes de lugares Apache: "ao se posicionar as mentes das pessoas a olhar 'para frente' (bidááh) dentro de um espaço, um nome de lugar também posiciona suas mentes a olhar 'para trás' ( $t$ 'zhi') no tempo".

Para as pessoas se imaginarem em pé na frente de um determinado lugar, elas precisam imaginar que estão em pé em trilhas de seus antepassados (biké'é nohwizá 'yé). Os antepassados também são fundamentais para o tempo e a vida Navajo, que segue uma linha de retorno curva em que "a conclusão gradual da vida de alguém é vista como um processo contínuo de crescimento de volta à ancestralidade; enquanto cresce e se torna mais velha, a pessoa muda continuamente, gradualmente, para se tornar um ancestral (que, no ocidente, poderíamos situar "no passado ')" (Pinxten, 1995: 240) (ver Yu, 2012, para uma discussão sobre esquematizações ancestrais em chinês e outras culturas).

Em terceiro lugar, enquanto a experiência do tempo é transcultural, o conceito autônomo e reificado de "Tempo como tal" é, como argumentado por Sinha et al. (2011), uma invenção histórica e cultural, não um domínio cognitivo universal. É somente quando este domínio é construído, através da organização calendárico-numérica de S-tempo, que emerge o mapeamento metafórico sistemático espaço-tempo. Podemos argumentar que Pacha é também uma construção histórica e cultural, o resultado de um processo diferente e divergente. Embora o raciocínio metafórico tenha sido fundamental para este processo de construção, ver o espaço-tempo andino como uma "interpretação espacial de tempo" impõe uma distorção eurocêntrica sobre as línguas e culturas indígenas.

\section{Resumo e direcionamentos futuros}

Com base na revisão da pesquisa, concluímos que:

1. A metáfora linguística e/ou fusão espaço-tempo são comuns nas lín- 
guas do mundo e são provavelmente motivadas por processos cognitivos universais;

2. No entanto, há evidências de que o mapeamento metafórico sistemático espaço-tempo não é universal na linguagem;

3. A motivação da estrutura linguística por processos cognitivos (mesmo tendo em conta que o último é supostamente universal) nunca é direta, mas sempre mediada por padrões e processos culturais materiais e simbólicos;

4. A variação do espaço e do tempo nas línguas está situada culturalmente em uma variação sociocultural sistemática e multi-nível, expressa em práticas e artefatos, bem como em sistemas de crenças;

5. A conceitualização do espaço e do tempo nas línguas é determinada tanto ou mais por visões de mundo compartilhadas em áreas cultural-geográficas amplas como por relações filogenéticas, produzindo padrões inter-linguísticos tais como pacha andina;

6. O espaço não é o único domínio de origem para a conceitualização linguística e cultural do tempo, o qual se baseia também em sistemas cosmológicos e de parentesco.

A pesquisa neste campo continuará a empregar modelos multi-metodológicos qualitativos e quantitativos e supomos que irá adicionar cada vez mais a arqueologia à lista de disciplinas representadas. A pesquisa deve gravar sistemas numéricos, cosmologia, material cultural relevante e práticas associadas, bem como a conceitualização linguística e gestual do espaço e do tempo. O principal desafio nos próximos anos será o de ir além da documentação e classificação da variação, para desenvolver uma explicação teórica que englobe tanto as causas como as restrições à variação.

\section{Referências}

Adelaar, Willem F. H., and Pieter C. Muysken. 2007. The Languages of the Andes. Cambridge: Cambridge University Press.

Arguedas, J.M. 2011. El manuscrito de Huarochirí. Bilingual version by José María Arguedas. Ed. J. I. Úzquiza González. Madrid: Biblioteca Nueva.

Basso, Keith H. 1988. "'Speaking with Names': Language and Landscape among the Western Apache.” Cultural Anthropology 3 (2):99-130, may 1988. Doi:10.1525/ can.1988.3.2.02a00010.

Bernárdez, Enrique. 2013. "On the Cultural Character of Metaphor: Some Reflections on Universality and Culture-Specificity in the Language and Cognition of Time, Especially in Amerindian Languages." Review of Cognitive Linguistics 11 (1) : 1-35. Doi:10.1075/rcl.11.1.01ber.

Birth, Kevin K. 2014. "The Vindolanda Timepiece: Time and Calendar Reckoning in Roman Britain.” Oxford Journal of Archaeology 33 (4):395-411. 
Bohnemeyer, Jürgen. 2009. "Temporal Anaphora in a Tenseless Language." The Expression of Time in Language, pp. 83-128.

.2002. The Grammar of Time Reference in Yukatek Maya. Munich, Lincom.

Boivin, Nicole. 2008. Material Cultures, Material Minds: The Impact of Things on Human Thought, Society, and Evolution. Cambridge; New York: Cambridge University Press.

Boroditsky, Lera. 2001. "Does Language Shape Thought?: Mandarin and English Speakers' Conceptions of Time." Cognitive Psychology 43 (1):1-22. Doi:10.1006/ cogp.2001.0748.

- 2000. "Metaphoric Structuring: Understanding Time through Spatial Metaphors.” Cognition 75 (1):1-28.

Bourdieu, P. 1977. Outline of a Theory of Practice. Transl. R. Nice. Cambridge: Cambridge University Press.

Brown, Penelope. 2012. "Time and Space in Tzeltal: Is the Future Uphill?" Frontiers in Psychology 3:212. Doi:10.3389/fpsyg.2012.00212.

Calvo Pérez, J. 1995. "Introducción a la lengua y cultura quechuas". Valencia: Universitat de València.

.Clark, A. 2006. "Language, Embodiment, and the Cognitive Niche." Trends in Cognitive Sciences 10 (8):370-74. Doi:10.1016/j.tics.2006.06.012.

Clark, H.H. 1973. "Space, time, semantics and the child". In Moore, T. E. (ed.) Cognitive Development and the Acquisition of Language. New York: Academic Press, pp. 27-63.

Casasanto, Daniel, and Lera Boroditsky. 2008. "Time in the Mind: Using Space to Think about Time.” Cognition 106(2):579-93. Doi:10.1016/ j.cognition.2007.03.004.

Dehaene, S., V. Izard, E. Spelke, and P. Pica. 2008. "Log or Linear? Distinct Intuitions of the Number Scale in Western and Amazonian Indigene Cultures." Science 320(5880):1217-20, 30 may 2008. Doi:10.1126/ science.1156540.

Einstein, A. 1920. "Relativity: The Special and the General Theory”. London: Methuen.

Denny, J. Peter. 1999. “Review.” Anthropological Linguistics 41(4):541-49.

Evans, Vyvyan. 2013. Language and Time: A Cognitive Linguistics Approach. Cambridge: Cambridge University Press.

Evans-Pritchard, E. E. 1939. "Nuer time-reckoning". Africa: Journal of the International African Institute 12(2):189-216.

Evans-Pritchard, E. E. Nuer. Oxford: Oxford University Press, 1940.

Fauconnier, Gilles, and Mark B. Turner. 2008. "Rethinking Metaphor.” SSRN Scholarly Paper. Rochester, NY: Social Science Research Network, http://papers.ssrn.com/ abstract $=1275662$.

Faller, M. and M. Cuéllar. 2003. Metáforas del tiempo en el quechua. (CD-ROM publication). 
Fedden, Sebastian, and Lera Boroditsky. 2012. "Spatialization of Time in Mian." Frontiers in Psychology 3. Doi:10.3389/fpsyg.2012.00485.

Flannery, Kent, and Joyce Marcus. 2012. The Creation of Inequality: How Our Prehistoric Ancestors Set the Stage for Monarchy, Slavery, and Empire. Cambridge: Harvard University Press.

Gaby, Alice. 2012. "The Thaayorre Think of Time Like They Talk of Space." Frontiers in Psychology 3. Doi:10.3389/fpsyg.2012.00300.

Gell, Alfred. 1992. The Anthropology of Time: Cultural Constructions of Temporal Maps and Images. Berg.

Goddard, C. 2010. "The natural semantic metalanguage approach". The Oxford Handbook of Linguistic Analysis. Oxford: Oxford University Press, pp. 459-484.

Haspelmath, Martin. 1997. From Space to Time: Temporal Adverbials in the World's Languages. Lincom Europa.

Hornborg, Alf, Rafael Gassn, Michael Heckenberger, Jonathan D Hill, Eduardo Ges Neves, Fernando Santos Granero, and Alf Hornborg. 2005. "Ethnogenesis, Regional Integration, and Ecology in Prehistoric Amazonia: Toward a System Perspective 1." Current Anthropology 46(4):589-620.

- 2005. "Ethnogenesis, Regional Integration, and Ecology in Prehistoric Amazonia: Toward a System Perspective 1." Current Anthropology 46 (4): 589-620.

Hurtado de Mendoza S., W. 2002. Pragmática de La Cultura Y La Lengua Quechua. Quito: Abya-Yala.

Hutchins, Edwin. 1996. Cognition in the Wild. New edition edition. Cambridge, Mass: MIT Press.

Keyes, Charles F. 1975. "Buddhist Pilgrimage Centers and the Twelve-Year Cycle: Northern Thai Moral Orders in Space and Time." History of Religions 15 (1):71-89.

Lakoff, George, and Mark Johnson. 1999. Philosophy in the Flesh: The Embodied Mind and Its Challenge to Western Thought. New Ed edition. New York, NY: Basic Books.

-1999. Philosophy in the Flesh: The Embodied Mind and Its Challenge to Western Thought. New Ed edition. New York, NY: Basic Books.

Lakoff, George, and Rafael E. Núñez. 2000. Where Mathematics Comes from: How the Embodied Mind Brings Mathematics into Being. 1st ed. New York, NY: Basic Books.

Lass, Andrew. 2012. “Objects of Time: How Things Shape Temporality. A1-Kevin K. Birth. New York: PB - Palgrave Macmillan, 212p. American Ethnologist 41(1):21920, 2014. Doi:10.1111/amet.12070_22.

Leavitt, J. 2010. Linguistic Relativities: Language diversity and modern thought. Cambridge: Cambridge University Press.

Lee, Penny. 1996. The Whorf Theory Complex: A Critical Reconstruction. Studies in the History of the Language Sciences. Amsterdam: John Benjamins Publishing Company. Vol. 81. http://www.jbe-platform.com/ content/books/9789027283900 
Le Guen, Olivier, and Lorena Ildefonsa Pool Balam. 2012. "No Metaphorical Timeline in Gesture and Cognition Among Yucatec Mayas." Frontiers in Psychology 3. Doi:10.3389/fpsyg.2012.00271.

Levine, Robert. 2006. A Geography of Time: The Temporal Misadventures of a Social Psychologist, or How Every Culture Keeps Time Just a Little Bit Differently. Oxford: Oneworld.

Levinson, Stephen C., and Asifa Majid. 2013. "The Island of Time: Yélî Dnye, the Language of Rossel Island." Frontiers in Psychology 4. Doi: 10.3389/ fpsyg.2013.00061.

Magnani, L. 2009. Abductive Cognition. The epistemological and eco-cognitive dimensions of hypothetical reasoning. Cognitive Systems Monographs 3. Berlin: Springer.

Malotki, Ekkehart. 1983. Hopi Time: A Linguistic Analysis of the Temporal Concepts in the Hopi Language. Walter de Gruyter.

McTaggart, J. 1908. The unreality of time. Mind: A Quarterly Review of Psychology and Philosophy 17:456-473.

Minkovsky, H. 1964. Space and Time. In Smart, J. J.C. (Ed.): Problems of Space and Time London: Macmillan.

Moore, Kevin Ezra. 2011. "Ego-Perspective and Field-Based Frames of Reference: Temporal Meanings of Front in Japanese, Wolof, and Aymara." Journal of Pragmatics 43:759-76. Doi:10.1016/j.pragma.2010.07.003.

.2006. "Space-to-time mappings and temporal concepts". Cognitive Linguistics 17: 199-244.

Newton, I.S. 1686. Philosophiæ Naturalis Principia Mathematica. London: Royal Society.

Nordlinger, Rachel, and Louisa Sadler. 2004. "Nominal Tense in Crosslinguistic Perspective." Language 80(4): 776-806.

Núñez, Rafael, and Kensy Cooperrider. 2013. "The Tangle of Space and Time in Human Cognition.” Trends in Cognitive Sciences 17(5): 220-29. Doi: 10.1016/j. tics.2013.03.008.

Núñez, Rafael, Kensy Cooperrider, D. Doan, and Jürg Wassmann. 2012. “Contours of Time: Topographic Construals of Past, Present, and Future in the Yupno Valley of Papua New Guinea.” Cognition 124(1):25-35. Doi: 10.1016/j.cognition.2012.03.007.

Núñez, Rafael E., and Carlos Cornejo. 2012. "Facing the Sunrise: Cultural Worldview Underlying Intrinsic-Based Encoding of Absolute Frames of Reference in Aymara." Cognitive Science 36(6):965-91. Doi: 10.1111/ j.1551-6709.2012.01237.x.

Nunez, Rafael E., and Eve Sweetser. 2006. "With the Future behind Them: Convergent Evidence from Aymara Language and Gesture in the Crosslinguistic Comparison of Spatial Construals of Time." Cognitive Science 30(3): 401-50.

Ohnuki-Tierney, Emiko. 1973. "Sakhalin Ainu Time Reckoning." Man, New Series, 8(2):285-99. Doi:10.2307/2800850. 
Pinxten, Rik. 1995. “Comparing Time and Temporality in Cultures.” Cultural Dynamics 7(2): 233-52. Doi:10.1177/092137409500700204.

Postill, John. 2002. "Clock and Calendar Time A Missing Anthropological Problem." Time \& Society 11(2-3): 251-70.

Radden, G. 2011. "Spatial time in the West and the East". In M. Brdar, M. Omazic, V. Pavicic Takac, T. Gradecak-Erdeljic, and G. Buljan (eds.) Space and Time in Language. Frankfurt: Peter Lang, pp. 1-40.

Reynoso, C. 2004. "Lenguaje y pensamiento: Tácticas y estrategias del relativismo linguístico" Unpublished manuscript, Universidad de Buenos Aires. Versión 13.04.04 Downloaded 07/05/2013 from http://carlosreynoso. com.ar.

Sampaio, W. and Silva Sinha, V. 2012. Dados Espaço-Tempo (Projeto Açai). Unpublished data archive.

Schieffelin, Bambi B. 2002. "Marking Time: The Dichotomizing Discourse of Multiple Temporalities." Current Anthropology 43(S4): 15-17. Doi: 10.1086/341107.

Silva Sinha, V. da, Sinha, C., Sampaio, W. and Zinken, J. 2103. "Event- based time intervals in an Amazonian culture". In Filipovič, L. and Kasia M. Jaszczolt (eds.) Space and Time across Languages and Cultures Vol. II Language, Culture and Cognition. Amsterdam: John Benjamins, pp. 15-35.

Silva Sinha, V. da and Sampaio, W. 2012. "Unpublished data corpus of space-time mappings in Amazonian languages".

Sinha, C. 2006. "Epigenetics, semiotics and the mysteries of the organism". Biological Theory 1(2):1-4.

2009. "Language as a biocultural niche and social institution". In Evans, V. \& S. Pourcel (eds.) New Directions in Cognitive Linguistics. Amsterdam: John Benjamins, pp. 289-310.

2013. "Niche construction, too, unifies praxis and symbolization". Language and Cognition 5:261-271.

Sinha, C. and Jensen de López, K. 2000. Language, culture and the embodiment of spatial cognition. Cognitive Linguistics 11:17-41.

Sinha, Chris, and Peter Gärdenfors. 2014. "Time, Space, and Events in Language and Cognition: A Comparative View." Annals of the New York Academy of Sciences 1326:72-81. Doi:10.1111/nyas.12491.

Sinha, Chris, Vera da Silva Sinha, Jörg Zinken, and Wany Sampaio. 2011. "When Time Is Not Space: The Social and Linguistic Construction of Time Intervals and Temporal Event Relations in an Amazonian Culture." Language and Cognition 3(1):137-69.

Smart, J.J.C. 1949. The River of Time. Mind. New Series 58(232):483-494.

Talmy, L. 1999. Fictive motion in language and "'ception". In: P. Bloom, M. Peterson, L. Nadel \& M. Garrett (eds.), Language and Space. Cambridge, MA: MIT Press, pp. 211-276. 
Whorf, B.L. 1950. An American Indian model of the universe. International Journal of American Linguistics 16:67-72.

Yu, Ning. 1998. The Contemporary Theory of Metaphor: A Perspective from Chinese.

Vol. 1. Human Cognitive Processing. Amsterdam: John Benjamins Publishing

Company. http://www.jbe-platform.com/content/books/9789027 282736.

. 2012. "The Metaphorical Orientation of Time in Chinese." Journal of Pragmatics 44(10):1335-54. Doi:10.1016/j.pragma.2012.06.002.

Data recebimento: $14 / 05 / 2015$

Data aceite: 15/07/2015 